\title{
Real time near-infrared Raman spectroscopy for the diagnosis of nasopharyngeal cancer
}

\author{
Lim Chwee Ming 1,2, Nagaraja Rao Gangodu ${ }^{1}$, Thomas Loh ${ }^{1,2}$, Wei Zheng ${ }^{3,4}$, Jianfeng \\ Wang $^{3}$, Kan Lin ${ }^{3,4}$ and Huang Zhiwei ${ }^{3}$ \\ ${ }^{1}$ Department of Otolaryngology-Head and Neck Surgery, National University Health System, Singapore \\ ${ }^{2}$ Division of Surgical Oncology, National Cancer Institute of Singapore, Singapore \\ ${ }^{3}$ Optical Bioimaging Laboratory, Department of Biomedical Engineering, Faculty of Engineering, National University of \\ Singapore, Singapore \\ ${ }^{4}$ Department of Medicine, Yong Loo Lin School of Medicine, National University of Singapore and National University Health \\ System, Singapore \\ Correspondence to: Lim Chwee Ming, email: Chwee_ming_lim@nuhs.edu.sg \\ Huang Zhiwei, email: biehzw@nus.edu.sg
}

Keywords: Raman spectroscopy, nasopharyngeal cancer, real time imaging, PLS-DA model, surveillance

Received: December 16, $2016 \quad$ Accepted: April 24, 2017 Published: May 08, 2017

Copyright: Ming et al. This is an open-access article distributed under the terms of the Creative Commons Attribution License 3.0 (CC BY 3.0 ), which permits unrestricted use, distribution, and reproduction in any medium, provided the original author and source are credited.

\section{ABSTRACT}

Near-infrared (NIR) Raman spectroscopy has been investigated as a tool to differentiate nasopharyngeal cancer (NPC) from normal nasopharyngeal tissue in an ex-vivo setting. Recently, we have miniaturized the fiber-optic Raman probe to investigate its utility in real time in-vivo surveillance of NPC patients. A posterior probability model using partial linear square (PLS) mathematical technique was constructed to verify the sensitivity and specificity of Raman spectroscopy in diagnosing NPC from post-irradiated and normal tissue using a diagnostic algorithm from three significant latent variables. NIR-Raman signals of 135 sites were measured from 79 patients with either newly diagnosed NPC $(N=12)$, post irradiated nasopharynx $(N=37)$ and normal nasopharynx $(N=30)$. The mean Raman spectra peaks identified differences at several Raman peaks at $853 \mathrm{~cm}^{-1}$, $940 \mathrm{~cm}^{-1}, 1078 \mathrm{~cm}^{-1}, 1335 \mathrm{~cm}^{-1}, 1554 \mathrm{~cm}^{-1}, 2885 \mathrm{~cm}^{-1}$ and $2940 \mathrm{~cm}^{-1}$ in the three different nasopharyngeal conditions. The sensitivity and specificity of distinguishing Raman signatures among normal nasopharynx versus NPC and post-irradiated nasopharynx versus NPC were $91 \%$ and $95 \%$; and $77 \%$ and $96 \%$ respectively. Real time near-infrared Raman spectroscopy has a high specificity in distinguishing malignant from normal nasopharyngeal tissue in vivo, and may be investigated as a novel non-invasive surveillance tool in patients with nasopharyngeal cancer.

\section{INTRODUCTION}

Non-keratinizing nasopharyngeal cancer (NPC) is a cancer that originates from the nasal epithelium of nasopharynx and it is often difficult to detect in the early stage due to the deep anatomical location. It is a common cancer in Asia where the incidence reaches its peak in countries such as Hong Kong, Southern China and Singapore. In Singapore, it is the 8th most common cancer among males [1] and carries with it risk of loco-regional recurrence of up to $20 \%$ following definitive chemoradiotherapy [2].
Early detection of local recurrence in NPC is pivotal towards successful surgical salvage, which has been shown to improve survival [3]. However, the challenge lies in having a reliable and accurate method of detecting early recurrence. Currently, the most reliable way of detecting nasopharyngeal recurrence is endoscopic examination of the nasopharynx, although the morphology of early recurrences is unknown. Most cases of recurrences are detected as a recurrent mass or ulcer in the nasopharynx. These recurrences are sometimes not surgically salvageable due to local extension of the tumor to critical structures such as the internal carotid artery, 
skull base and optic nerve that are in close proximity to the nasopharynx. Therefore, identifying early morphologic changes preceding the development of these clinically obvious recurrences will allow prompt surgical salvage of these recurrences; and hence improve clinical outcomes of NPC patients.

Raman spectroscopy is a unique optical technique, which utilizes inelastic scattering of light from tissue to characterize its molecules and tissue composition. This system has been well studied in gastric cancer in diagnosing dysplasia and premalignant condition [4-6]. Lau DP et al. reported a preliminary analysis on differentiating nasopharyngeal cancer from normal tissue ex vivo using Raman spectroscopy [7]. However, the data was not optimized as it only covered a narrow spectral window of $950-1650 \mathrm{~cm}^{-1}$ with no statistical significance. Recently, Huang's group [8] has developed a rapid fiber-optic NIR Raman spectroscopy system for better characterization of tissue Raman signals in vivo, and miniaturizing the probe size to $1.8 \mathrm{~mm}$; which allows clinicians to obtain a more reliable Raman signals in tight anatomical confines in the nasopharynx.

Putting this together, we aim to test this new NIR Raman spectroscopy method in detecting distinctive molecular signatures specific to normal healthy nasopharynx, post-irradiated nasopharynx and NPC tissue. The clinical relevance of this study is to investigate if this tool may be employed in the surveillance of NPC patients; with the overarching goal to detect early Raman signals which precede the development of clinically apparent local recurrence in the nasopharynx of these patients.

\section{RESULTS}

Raman signatures $(N=135)$ were obtained from 79 patients (12 NPC; 30 normal patients and 37 postirradiated) and the patients' characteristics are summarized in Table 1. When the cumulated Raman spectra peaks of these patients were analyzed, distinct differences is mean amplitudes in Raman peaks were identified at $853 \mathrm{~cm}^{-1}$, $940 \mathrm{~cm}^{-1}, 1078 \mathrm{~cm}^{-1}, 1335 \mathrm{~cm}^{-1}, 1554 \mathrm{~cm}^{-1}, 2885 \mathrm{~cm}^{-1}$ and $2940 \mathrm{~cm}^{-1}$ among patients with NPC, normal nasopharynx and post-irradiated nasopharynx (Figure 1).

Using partial least squares regression method, comparison of collective Raman signatures between normal nasopharynx versus post-irradiated nasopharynx (Figure 2); NPC versus normal nasopharynx (Figure 3); and NPC versus post-irradiated nasopharynx were constructed (Figure 4). The corresponding sensitivity and specificity for the respective paired cohorts were post-irradiated nasopharynx versus normal nasopharynx (sensitivity $80 \%$; specificity $88 \%$ ); normal nasopharynx versus NPC (sensitivity 91\%; specificity 95\%); and lastly between post irradiated nasopharynx versus NPC(sensitivity 77\%; specificity 96\%).

\section{DISCUSSION}

Utilizing Raman spectroscopy in cancer diagnostics has gained momentum over the past decade. This technology has been used in the differentiating normal and cancerous tissues in lung cancer [9, 10], gastric cancer [6, 11, 12], cervical cancer [13], laryngeal and nasopharyngeal cancer [7, 14-16]. Much of this enthusiasm lies in the capability and reliability of Raman scattering signals to reveal biomedical and bio-molecular signatures that are unique to the tissue composition being evaluated and hence, provide an opportunity to distinguish different pathologies on tissues at a molecular level.

In this pilot study, we sought to examine the Raman signatures in 3 patient cohorts, namely in patients with nasopharyngeal cancer, normal nasopharynx and post-irradiated nasopharynx following radiotherapy for nasopharyngeal cancer. The main motivation in

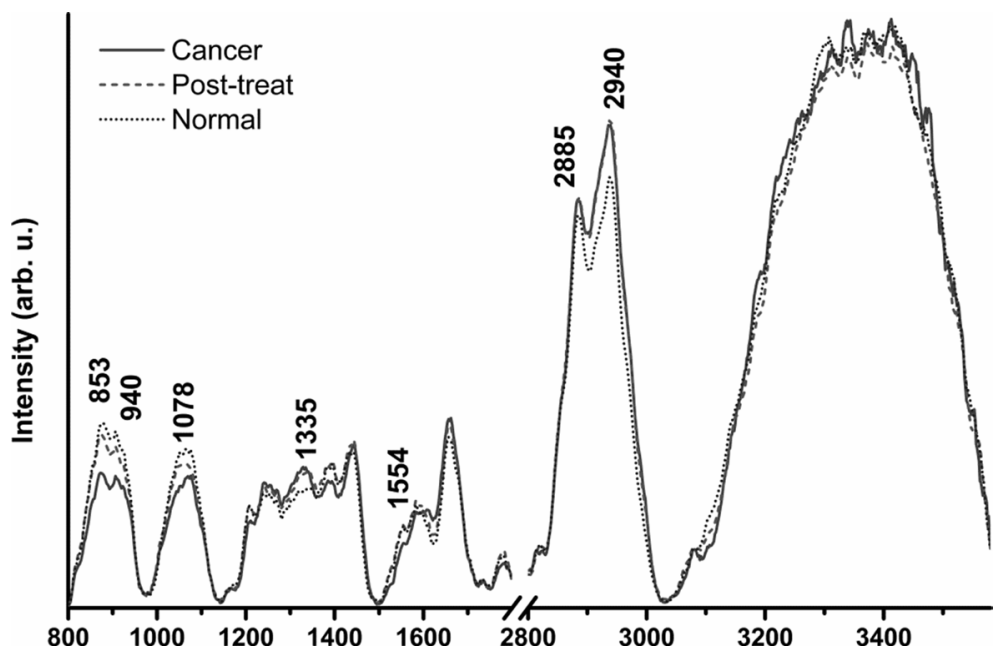

Figure 1: Raman Spectra peaks for patients with normal nasopharynx, post irradiated nasopharynx and nasopharyngeal cancer. 
Table 1: Summary of patient's characteristics

\begin{tabular}{cccc}
\hline Category & Normal Nasopharynx & $\begin{array}{c}\text { Post-Irradiated } \\
\text { Nasopharynx }\end{array}$ & Nasopharyngeal cancer \\
\hline $\begin{array}{c}\text { Number of patients }(\boldsymbol{n}=\mathbf{7 9}) \\
\text { Number of collected sites } \\
\text { data }(\boldsymbol{n}=\mathbf{1 3 5})\end{array}$ & 30 & 37 & 12 \\
Mean Age (S.D.) & 42 & 71 & 22 \\
Gender & $54.4(18.9)$ & $52.9(12.3)$ & $52.6(11.7)$ \\
Stage & 23 Males/ & 27 Males/ & $6 \mathrm{Males} /$ \\
& 7 Females & N females & 6 Females \\
\hline
\end{tabular}

performing this pilot study is to establish if a differential Raman scattering signatures may be obtained reliably using a $1.8 \mathrm{~mm}$ probe, which is currently the smallest probe utilized towards Raman spectroscopy diagnostics. With this validation study, the possibility of using Raman spectroscopy may be envisaged in earlier diagnosis of local recurrence of nasopharyngeal cancer; which is usually detected late given the deep anatomical location.

Our real-time in-vivo test using this fiber-opticRaman $(1.8 \mathrm{~mm})$ probe was successful in obtaining reliable and consistent Raman signatures among patients with nasopharyngeal cancer, post-irradiated nasopharynx and normal controls. There were several Raman spectra peaks whereby distinct Raman scattering peaks were obtained and hence, validated our hypothesis that these tissues exhibited different tissue compositions, which can be picked up by Raman spectroscopy. We have adopted the PLS algorithm in constructing our normogram data based on our previous published reports $[17,18]$ as well as a recent study in nasopharyngeal cancer, where the PLS algorithm was more sensitive than the PCA model in detecting malignant Raman signatures in nasopharyngeal cancer [19]. Additionally, the PLS regression method further rotates the components (latent variables (LVs)) to achieve the maximum group separation compared to the PCA model. Hence, these LVs could explain the diagnostic relevant variations rather than the significant differences in the dataset.

We believe that our study represents a step forward in the development of Raman spectroscopy in the diagnostic of nasopharyngeal cancer on several counts. Firstly, ever since the first report of using Raman spectroscopy in nasopharyngeal cancer by Lau et al. [7], all previous studies have been based on using Raman signatures taken on ex-vivo nasopharyngeal tissues which may result in differences in the Raman signatures due to desiccation efforts following tissue ex-plantation $[15,19,20]$. Therefore, the clinical utility of real-time molecular diagnostics using Raman spectroscopy cannot be directly translated. Secondly, to our knowledge, the $1.8 \mathrm{~mm}$ probe size is the smallest

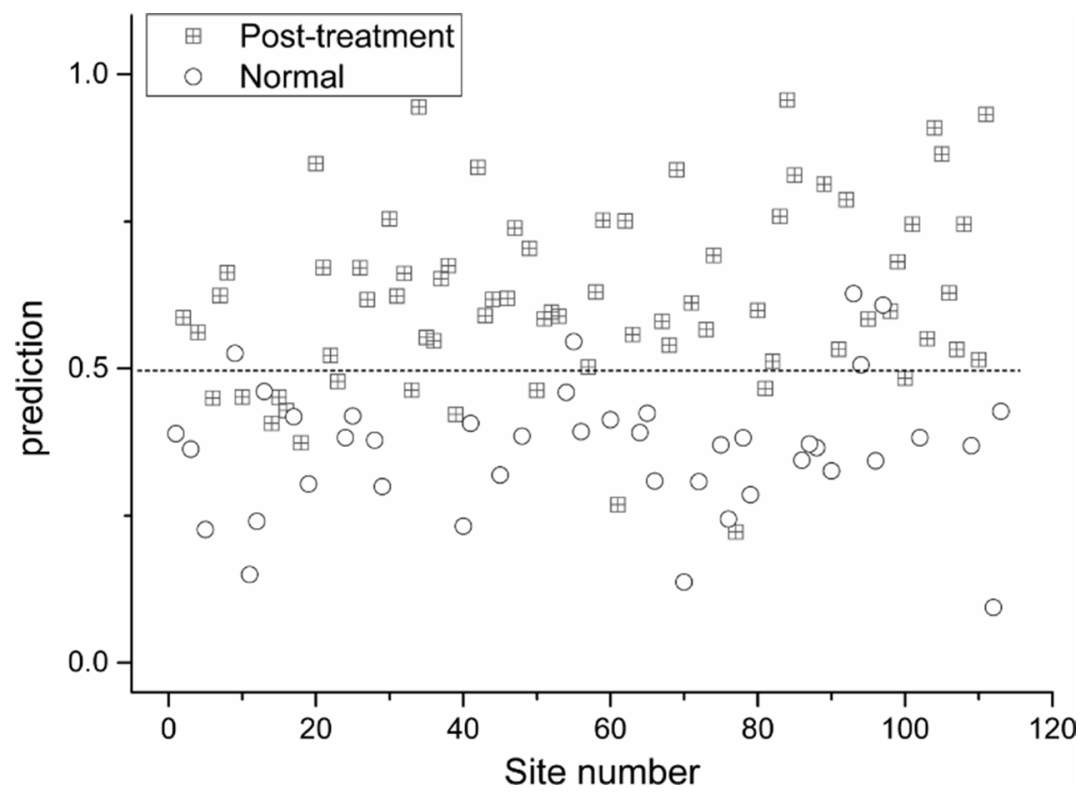

Figure 2: Scatter plot of the posterior probability model using partial linear square technique of patients with normal nasopharynx versus post-irradiated nasopharynx (sensitivity $80 \%$; specificity $88 \%$ ). 
size being used for evaluation in Raman spectroscopy. Li et al. [15] performed micro-Raman spectroscopy on tissue samples in differentiating the Raman signatures between normal nasopharyngeal tissue and nasopharyngeal cancer. The micro-Raman tissue measurements based on commercially bulky Raman microscope system are impractical for clinical Raman applications at endoscopy. Therefore, a smaller caliber probe is more desirable.
Lastly, we have included an additional cohort of patients following radiotherapy of their nasopharyngeal cancer. In this regard, Raman signatures among patients with post-irradiated nasopharynx may be investigated and studied so that any Raman shifts between post-irradiated nasopharynx and nasopharyngeal cancer may be analyzed. This has translational application towards surveillance of the nasopharynx for recurrence following radiotherapy.

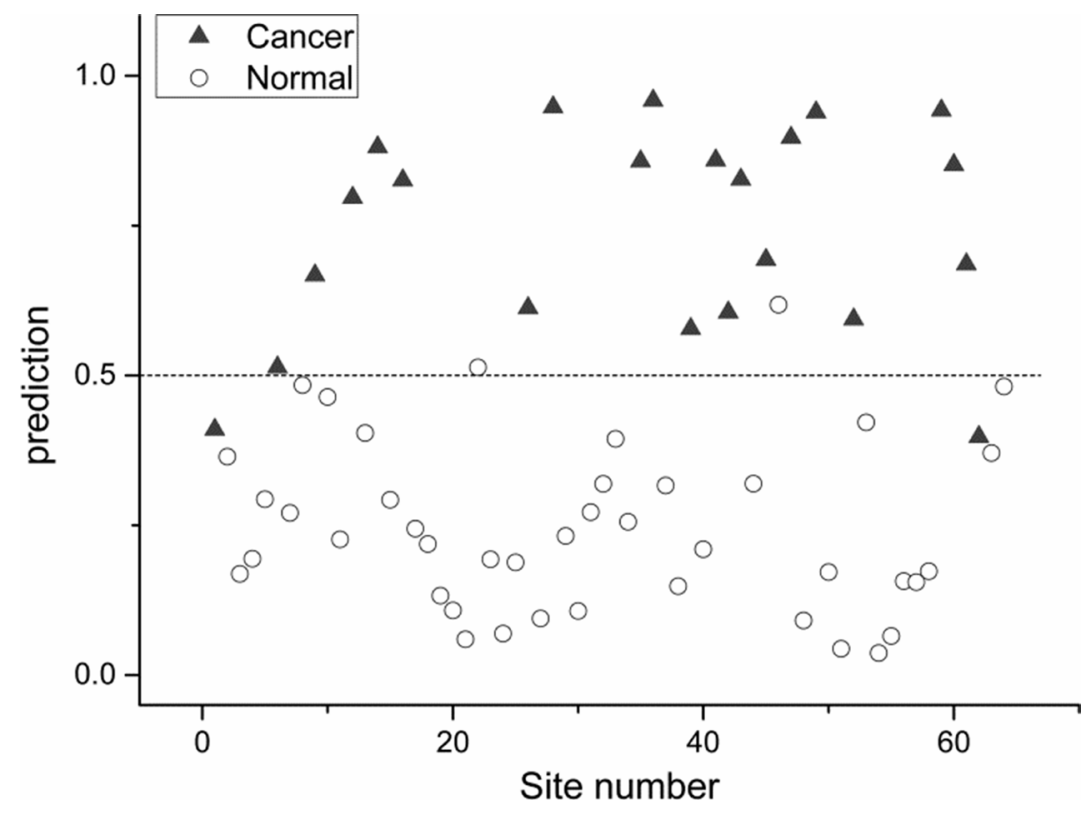

Figure 3: Scatter plot of the posterior probability model using partial linear square technique of patients with normal nasopharynx versus nasopharyngeal cancer (sensitivity $91 \%$; specificity $95 \%$ ).

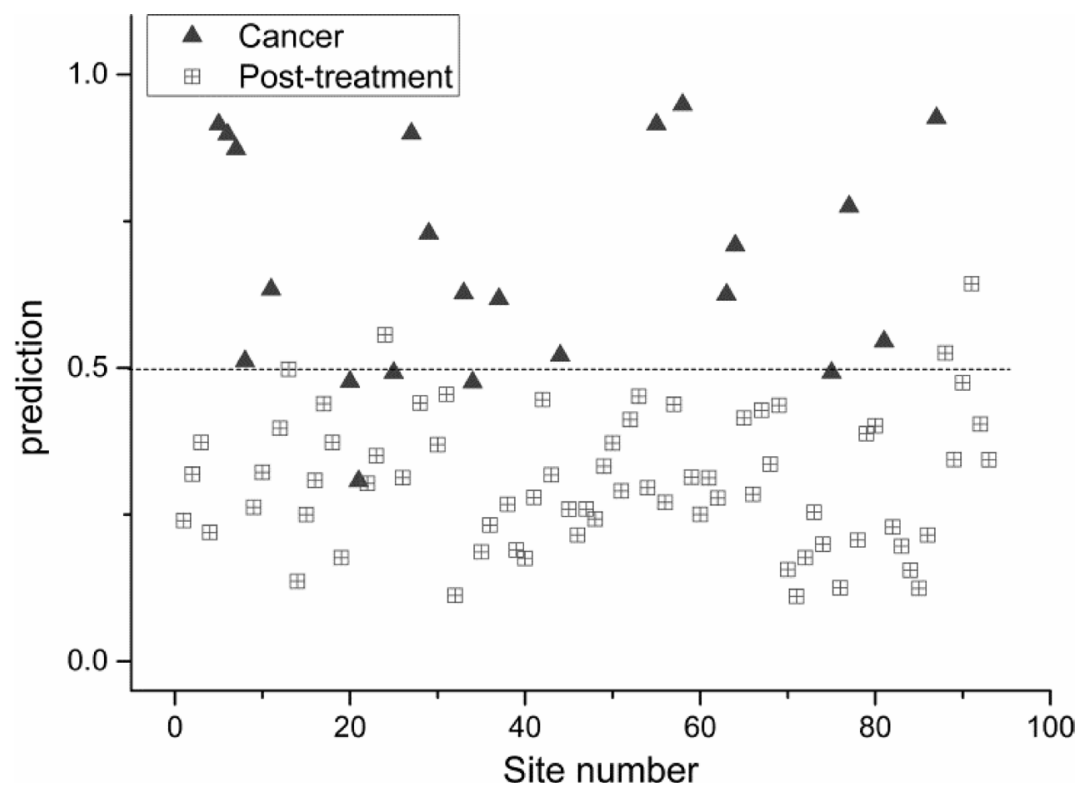

Figure 4: Scatter plot of the posterior probability model using partial linear square technique of patients with postirradiated nasopharynx versus nasopharyngeal cancer (sensitivity $77 \%$; specificity $96 \%$ ). 
Our pilot study demonstrated a relatively high specificity of $95 \%$ and $96 \%$ in distinguishing normal nasopharynx versus nasopharyngeal cancer and postirradiated nasopharynx versus nasopharyngeal cancer. With this high specificity values, we believe our in vivo fiber-optic Raman spectroscopy system has the potential for real time surveillance of nasopharyngeal cancer by selecting patients with "malignant" Raman signatures for further confirmation by histology. Additionally, patients with previously treated nasopharyngeal cancer who present with a normal looking nasopharynx plus a "benign" Raman signature may be more reassured of having no local disease; rather than relying on a purely normal looking nasopharyngeal finding on endoscopy.

We demonstrate for the first time that real-time near-infrared Raman spectroscopy using a $1.8 \mathrm{~mm}$ fiberoptic Raman probe can detect reliable and consistent Raman scattering signatures among patients with nasopharyngeal cancer, post-irradiated nasopharynx and normal nasopharynx. The high specificity in distinguishing malignant from normal nasopharyngeal tissue and postirradiated nasopharyngeal tissue warrants investigation of this tool as a new non-invasive surveillance tool in patients with nasopharyngeal cancer.

\section{MATERIALS AND METHODS}

Under an Institutional approved clinical protocol (National Healthcare Group DSRB; 2014/00323), prospective patients with newly diagnosed NPC, postirradiated nasopharynx (at least 6 months following completion of radiotherapy) and normal nasopharynx were accrued in this study. Patients with nasopharyngeal cancer were biopsied proven to be cancer while patients with normal nasopharynx were identified based on normal endoscopic examination of the nasopharynx. Patients with post-irradiated nasopharynx were those who had received prior definitive radiotherapy for nasopharyngeal cancer and were disease free at the time of examination. This assessment was based endoscopic examination of the nasopharynx showing no residual tumor as well as a post-treatment magnetic resonance imaging (MRI) scan of the nasopharynx demonstrating complete resolution of their initial tumor. Any clinically suspicious areas of the nasopharynx were biopsied and were all proven to be negative of cancer. Additionally, all these patients with post-irradiated nasopharynx were followed up for at least 6 months with no evidence of recurrence.

\section{Detection of Raman signals of the nasopharynx}

During endoscopic examination of the nasopharynx, a1.8-mm miniature Raman probe is navigated to the nasopharynx under direct vision from the endoscope. In patients with normal and post irradiated nasopharynx, the probe is placed close to the fossa of Rosenmuller and additional measurements were performed in the midline of the nasopharynx of some patients (Figure 5). In patients with newly diagnosed (pre-treatment) NPC, the probe is positioned directly on the tumor (Figure 5). Once contact to the nasopharynx is achieved, a NIR

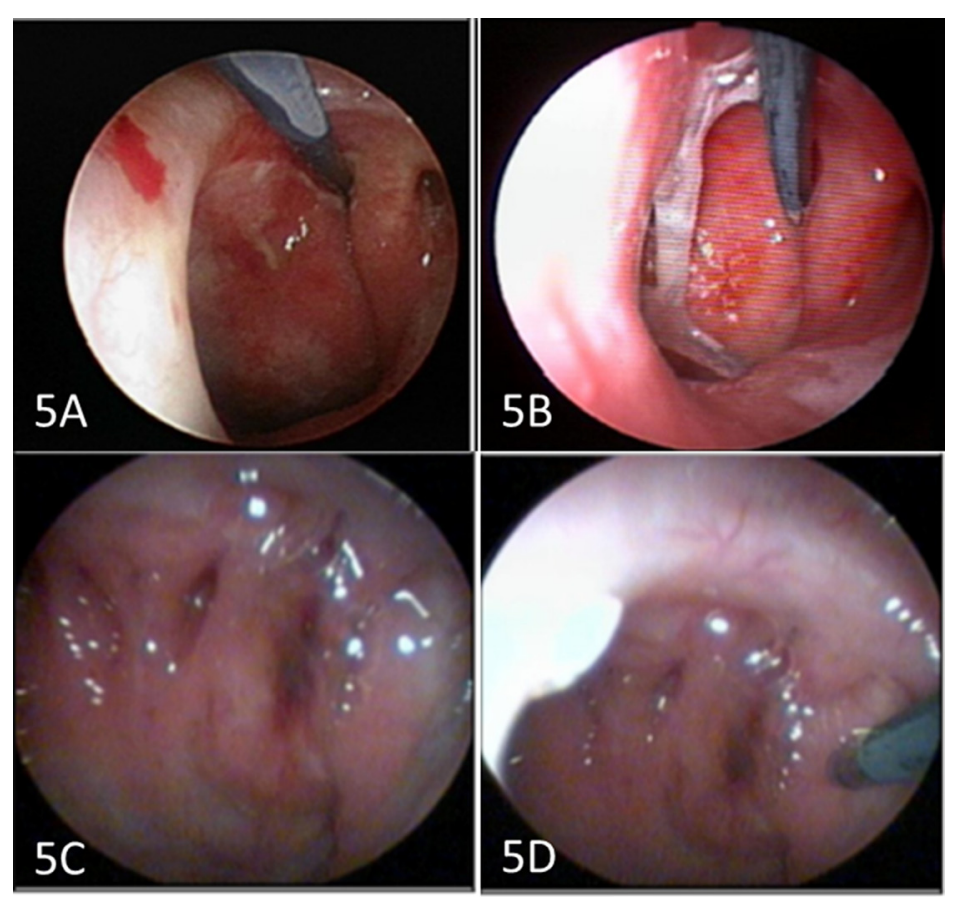

Figure 5: Endoscopic view of the micro-Raman probe in contact with nasopharynx- post-irradiated (A, B) and nasopharyngeal cancer $(\mathbf{C}, \mathbf{D})$. 
laser is emitted at 785-nm from the probe and the backscattered light (Raman signal) is captured by the Raman system. In this study, we have adopted a simultaneous fingerprint (FP) and high wavenumber (HW) fiberoptic Raman spectroscopy technique for real-time in vivo tissue Raman measurements during endoscopy. The 785-nm laser excitation power at $\sim 12 \mathrm{~mW}$ was selected as it was within the maximal permissible skin exposure limit set out for a 785-nm laser beam (American National Standards Institute). Multiple spectra ( 8-10) for each tissue site were measured with scanning times of 0.1 to $0.5 \mathrm{sec}$, which permits a rapid survey of the tissue areas. The entire process of capturing of Raman signals is typically completed within $15-20$ seconds.

\section{Construction of the nomogram data}

The NIR Raman spectra measured from in-vivo nasopharyngeal tissue represent a combination of weak tissue Raman signal, intense auto-fluorescence (AF) background and noise. The raw spectra are preprocessed by a third-order Savitzky-Golay smoothing filter (a window width of 3 pixels)to remove the spectral noise $[8,14,17,18]$. In the finger-print (FP) region (800-1800 $\left.\mathrm{cm}^{-1}\right)$, a fifth-order polynomial was found to be optimal for fitting the AF background in the noise-smoothed spectrum, and this polynomial was then subtracted from the measured FP spectrum to yield the FP tissue Raman spectrum alone. In the high-wave number (HW) range (2800-3600 $\mathrm{cm}^{-1}$ ), a first-order polynomial fit was used for removing the AF background [17, 21, 22]. The FP/HW Raman spectra are then normalized over the integrated area under the FP and HW ranges to allow a better comparison of the spectral shapes and relative Raman band intensities among NPC, normal and post-irradiated tissues.

Partial least squares (PLS) (also called Projection to Latent Structure) regression is a validated method for modeling in Raman system, providing an alternate approach to PCA (Principle component analysis). PLS technique is an extension of the multiple linear regression model and a linear model specifies the (linear) relationship between a dependent (response) variable Y, and a set of predictor variables, the X's. It follows the principle of PCA, but further rotates the components (latent variables (LVs)) to achieve the maximum group separation. Hence, the LVs could explain the diagnostic relevant variations rather than the significant differences in the dataset. Many studies have constructed and validated this statistical model to predict the sensitivity and specificity of Raman system in NPC model [15, 23, 24]. Additionally, consistent and reproducible results using this statistical model have been validated in various cancers including, gastric cancer $[11,12]$, esophageal cancer $[11,25]$, colon cancer $[21,26]$ and NPC [14].

\section{Abbreviations}

NPC- Nasopharyngeal carcinoma, PLS-DA -Partial Least Square- Discriminant Analysis, FP- Finger Print, HW- High Wave number, NIR-Near Infra-Red, AF- Auto florescence.

\section{Authors' contributions}

Dr Lim Chwee Ming has significantly contributed to the article by writing, editing and reviewing it. Dr Huang Zhi Wei and the co-authors have contributed for the instrumentation development, data collection and analysis, editing and reviewing this paper.

\section{CONFLICTS OF INTEREST}

The author and co-authors have no conflicts of interest to declare.

\section{FUNDING}

Dr Chwee Ming Lim acknowledges the funding support from the National Medical Research Council Singapore (Transitional Award, 0035/2015; NMRC/ $\mathrm{BnB} / 2015$ ) and Seed Grant from the National University Cancer Institute Singapore. Dr Huang Zhiwei acknowledges the funding support from the National Medical Research Council (NMRC) (CIRG/1331/2012; NMRC/BnB/0012b/2014; NMRC/BnB/0012c/2014; NMRC/TCR/016-NNI/2016), and the Academic Research Fund (AcRF)-Tier 2 from Ministry of Education (MOE) (MOE2014-T2-1-010), Singapore.

\section{REFERENCES}

1. Singapore Cancer Registry. Interim Annual Report.Trends in cancer incidence in Singapore 2010-2014. (2015). Retrieved 20 April 2016, from National Regsitry of Diseases office https://www.nrdo.gov.sg/docs/librariesprovider3/ default-document-library/cancer-trends-2010-2014_interimannual-report_final-(public).pdf?sfvrsn $=0$.

2. Mak HW, Lee SH, Chee J, Tham I, Goh BC, Chao SS, Ong YK, Loh KS, Lim CM. Clinical Outcome among Nasopharyngeal Cancer Patients in a Multi-Ethnic Society in Singapore. PloS one. 2015; 10:e0126108. doi:10.1371/ journal.pone.0126108.

3. Na'ara S, Amit M, Billan S, Cohen JT, Gil Z. Outcome of patients undergoing salvage surgery for recurrent nasopharyngeal carcinoma: a meta-analysis. Annals of surgical oncology. 2014; 21:3056-62. doi:10.1245/s10434-014-3683-9.

4. Bergholt MS, Zheng W, Lin K, Ho KY, Teh M, Yeoh KG, So JB, Huang Z. Combining near-infrared-excited autofluorescence and Raman spectroscopy improves in vivo 
diagnosis of gastric cancer. Biosensors \& bioelectronics. 2011; 26:4104-10. doi:10.1016/j.bios.2011.04.005.

5. Huang Z, Bergholt MS, Zheng W, Lin K, Ho KY, Teh M, Yeoh KG. In vivo early diagnosis of gastric dysplasia using narrow-band image-guided Raman endoscopy. Journal of biomedical optics. 2010; 15:037017. doi:10.1117/1.3420115.

6. Wang J, Lin K, Zheng W, Ho KY, Teh M, Yeoh KG, Huang Z. Comparative study of the endoscope-based bevelled and volume fiber-optic Raman probes for optical diagnosis of gastric dysplasia in vivo at endoscopy. Analytical and bioanalytical chemistry. 2015; 407:8303-10. doi:10.1007/s00216-015-8727-x.

7. Lau DP, Huang Z, Lui H, Man CS, Berean K, Morrison MD, Zeng H. Raman spectroscopy for optical diagnosis in normal and cancerous tissue of the nasopharynx-preliminary findings. Lasers in surgery and medicine. $2003 ; 32: 210-4$. doi:10.1002/1sm.10084

8. Huang Z, Teh SK, Zheng W, Mo J, Lin K, Shao X, Ho KY, Teh M, Yeoh KG. Integrated Raman spectroscopy and trimodal wide-field imaging techniques for real-time in vivo tissue Raman measurements at endoscopy. Optics letters. 2009; 34:758-60

9. Huang Z, McWilliams A, Lui H, McLean DI, Lam S, Zeng H. Near-infrared Raman spectroscopy for optical diagnosis of lung cancer. International journal of cancer. 2003; 107:1047-52. doi:10.1002/ijc.11500.

10. Huang Z, McWilliams A, Lam S, English J, McLean DI, Lui H, Zeng H. Effect of formalin fixation on the nearinfrared Raman spectroscopy of normal and cancerous human bronchial tissues. International journal of oncology. 2003; 23:649-55

11. Bergholt MS, Zheng W, Lin K, Ho KY, Teh M, Yeoh $\mathrm{KG}$, So JB, Huang Z. Characterizing variability in in vivo Raman spectra of different anatomical locations in the upper gastrointestinal tract toward cancer detection. Journal of biomedical optics. 2011; 16:037003. doi:10.1117/1.3556723.

12. Duraipandian S, Sylvest Bergholt M, Zheng W, Yu Ho K, Teh M, Guan Yeoh K, Bok Yan So J, Shabbir A, Huang Z. Real-time Raman spectroscopy for in vivo, online gastric cancer diagnosis during clinical endoscopic examination. Journal of biomedical optics. 2012; 17:081418. doi:10.1117/1.jbo.17.8.081418.

13. Duraipandian S, Zheng W, Ng J, Low JJ, Ilancheran A, Huang Z. In vivo diagnosis of cervical precancer using Raman spectroscopy and genetic algorithm techniques. The Analyst. 2011; 136:4328-36. doi:10.1039/c1an15296c.

14. Bergholt MS, Lin $\mathrm{K}$, Zheng $\mathrm{W}$, Lau DP, Huang Z. In vivo, real-time, transnasal, image-guided Raman endoscopy: defining spectral properties in the nasopharynx and larynx. Journal of biomedical optics. 2012; 17:077002. doi:10.1117/1.jbo.17.7.077002.

15. Li Y, Pan J, Chen G, Li C, Lin S, Shao Y, Feng S, Huang Z, Xie S, Zeng H, Chen R. Micro-Raman spectroscopy study of cancerous and normal nasopharyngeal tissues. Journal of biomedical optics. 2013; 18:27003. doi:10.1117/1. jbo.18.2.027003.

16. Teh SK, Zheng W, Lau DP, Huang Z. Spectroscopic diagnosis of laryngeal carcinoma using near-infrared Raman spectroscopy and random recursive partitioning ensemble techniques. The Analyst. 2009; 134:1232-9. doi:10.1039/ b811008e.

17. Lin K, Cheng DL, Huang Z. Optical diagnosis of laryngeal cancer using high wavenumber Raman spectroscopy. Biosensors \& bioelectronics. 2012; 35:213-7. doi:10.1016/j. bios.2012.02.050.

18. Lin K, Wang J, Zheng W, Ho KY, Teh M, Yeoh KG, Huang Z. Rapid Fiber-optic Raman Spectroscopy for RealTime In Vivo Detection of Gastric Intestinal Metaplasia during Clinical Gastroscopy. Cancer prevention research (Philadelphia, Pa). 2016; 9:476-83. doi:10.1158/1940-6207. capr-15-0213.

19. Li Y, Huang W, Pan J, Ye Q, Lin S, Feng S, Xie S, Zeng H, Chen R. Rapid detection of nasopharyngeal cancer using Raman spectroscopy and multivariate statistical analysis. Molecular and clinical oncology. 2015; 3:375-80. doi:10.3892/mco.2014.473.

20. Bergholt MS, Duraipandian S, Zheng W, Huang Z. Multivariate reference technique for quantitative analysis of fiber-optic tissue Raman spectroscopy. Analytical chemistry. 2013; 85:11297-303. doi:10.1021/ac402059v

21. Bergholt MS, Lin K, Wang J, Zheng W, Xu H, Huang Q, Ren JL, Ho KY, Teh M, Srivastava S, Wong B, Yeoh KG, Huang Z. Simultaneous fingerprint and high-wavenumber fiber-optic Raman spectroscopy enhances real-time in vivo diagnosis of adenomatous polyps during colonoscopy. Journal of biophotonics. 2016; 9:333-42. doi:10.1002/ jbio.201400141.

22. Bergholt MS, Zheng W, Huang Z. Development of a multiplexing fingerprint and high wavenumber Raman spectroscopy technique for real-time in vivo tissue Raman measurements at endoscopy. Journal of biomedical optics. 2013; 18:030502. doi:10.1117/1. jbo.18.3.030502.

23. Qiu S, Huang Q, Huang L, Lin J, Lu J, Lin D, Cao G, Chen C, Pan J, Chen R. Label-free discrimination of different stage nasopharyngeal carcinoma tissue based on Raman spectroscopy. Oncology letters. 2016; 11:2590-94. doi:10.3892/ol.2016.4239.

24. Sun L, Xu Z, Huang W, Wu S, Lin X, Zhu F, Liu N, Huang M, Chen R, Zeng H. Preliminary study of differentiating smears from cancerous and non-cancerous nasopharyngeal tissue using confocal Raman spectroscopy. Journal of cancer research and clinical oncology. 2016; 142:823-31. doi:10.1007/s00432-015-2082-3.

25. Bergholt MS, Zheng W, Ho KY, Teh M, Yeoh KG, Yan So JB, Shabbir A, Huang Z. Fiberoptic confocal raman spectroscopy for real-time in vivo diagnosis of dysplasia in Barrett's esophagus. Gastroenterology. 2014; 146:27-32. doi:10.1053/j.gastro.2013.11.002. 
26. Bergholt MS, Zheng W, Lin K, Wang J, Xu H, Ren JL, Ho KY, Teh M, Yeoh KG, Huang Z. Characterizing variability of in vivo Raman spectroscopic properties of different anatomical sites of normal colorectal tissue towards cancer diagnosis at colonoscopy. Analytical chemistry. 2015; 87:960-6. doi:10.1021/ac503287u. 